\title{
ANÁLISE DE DESEMPENHO DE UMA REDE CELULAR CDMA ATRAVÉS DE SIMULAÇÃO
}

\author{
Luiz Gustavo Alarcon Alegro e Celso de Almeida \\ Departamento de Comunicações \\ Faculdade de Engenharia Elétrica e de Computação - UNICAMP \\ Caixa Postal 6101 - 13.081-970 - Campinas - S.P. - Brasil \\ celso@decom.fee.unicamp.br
}

\begin{abstract}
Resumo - A influência de alguns parâmetros sobre o desempenho de uma rede celular CDMA foi avaliada por meio de simulação, tais como: número de usuários em serviço, percentual de queda e de bloqueio de chamadas, raio da região de cobertura, percentual de usuários em processo de "handoff", e da probabilidade de perda de desempenho ("outage probability").
\end{abstract}

\begin{abstract}
The effects of some important parameters on the performance of a CDMA cellular network are examined. The performance is obtained by analyzing the number of users in the system, the blocking and dropped calls rate, the coverage radius, the soft-handoff rate, and the outage probability.
\end{abstract}

Palavras-chave: Rede Celular CDMA, Percentual de Bloqueio e de Queda de Chamadas, Região de Cobertura, Percentual de Usuários em Soft-Handoff, Probabilidade de Perda de Desempenho.

\section{INTRODUÇÃO}

O uso da técnica de múltiplo acesso por divisão por código (CDMA - "Code Division Multiple Access") tem sido investigada em uma enorme variedade de sistemas móveis celulares e de comunicação pessoal sem fio [1].

O desempenho de uma rede celular CDMA em termos da capacidade (número de usuários em serviço), do percentual de bloqueio e de queda de chamadas, e do percentual de usuários em processo de "handoff" foi obtido em [2], [3] em função da taxa de chegada de chamadas. Neste artigo, estes e outros parâmetros foram analisados, tais como, raio da região de cobertura, e probabilidade de perda de desempenho [4]. Além disso, os elementos de desempenho descritos anteriormente foram também analisados pela variação de alguns novos parâmetros, tais como, desvio padrão do sombreamento, fator de atividade de voz, e expoente da perda de propagação [4].

\section{MODELO DE SIMULAÇÃO}

O processo de chegada de chamadas em um sistema telefônico é modelado segundo uma distribuição de Poisson com taxa de chegada de chamadas igual a $\lambda$ usuários/s, e com intervalo entre chegadas obedecendo a uma distribuição exponencial negativa. A duração de cada chamada segue também uma distribuição exponencial negativa com duração média igual a $\mu \mathrm{s}$.
As estações rádio-móveis (ERM) são distribuídos uniformemente em um círculo, em cujo centro encontra-se uma estação rádio-base (ERB). Como conseqüência, temos que uma determinada ERM deve apresentar coordenadas polares dadas por:

$$
\begin{aligned}
r & =R \sqrt{u_{1}} \\
\theta & =2 \pi u_{2}
\end{aligned}
$$

onde $r$ representa a distância do usuário móvel ao centro da célula, $\theta$ ø ângulo medido no sentido anti-horário a partir do eixo das abcissas, $R$ é o raio da célula, $u_{1}$ e $u_{2}$ são variáveis aleatórias independentes uniformemente distribuídas entre 0 e 1. Cada ERM possui velocidade $v$ e direção $\phi$ iniciais modeladas por:

$$
\begin{aligned}
v & =\left(v_{\max }-v_{\min }\right) u_{3}+v_{\min } \\
\phi & =2 \pi u_{4}
\end{aligned}
$$

onde $v_{\max }$ e $v_{\min }$ representam as velocidades máxima e mínima, respectivamente, e $u_{3}$ e $u_{4}$ também são variáveis aleatórias independentes uniformemente distribuídas entre 0 e 1.

Dois fatores serão considerados no enlace rádio, a perda de propagação entre a ERB e a ERM, e também o sombreamento, tanto do enlace direto, quanto do reverso. O modelo de perda de propagação está baseado no crescimento exponencial da perda de percurso $L$ com a distância $r$, ou seja,

$$
L=\frac{P}{S}=r^{\beta}
$$

onde $P$ e $S$ são as potências médias transmitida e recebida, respectivamente. Diferentes padrões de agrupamentos urbanos, suburbanos e rurais podem ser modelados através da escolha apropriada do expoente de perda de propagação $\beta$. O sombreamento é modelado através de uma variável lognormal com média de $0 \mathrm{~dB}$ e desvio padrão de $\sigma \mathrm{dB}$. A cada $s$ metros consideraremos que os valores de sombreamento são descorrelacionados. Para os valores intermediários será feita uma interpolação linear. Neste trabalho usaremos $s=30 \mathrm{~m}$.

A atividade vocal é modelada por uma distribuição de Bernoulli, onde a probabilidade de uma determinada ERM encontrar-se em silêncio é dada por $1-\nu$, enquanto que a probabilidade desta se encontrar em atividade vocal é dada por $\nu$.

O controle de potência no canal direto não é de domínio público, cabendo aos fabricantes de equipamentos desenvolver o seu próprio algoritmo. É suposto que no canal direto a 
ERM faz uma estimativa da perda de propagação, através do piloto, bem como da intensidade de interferência à qual ela está sendo submetida, e as envia para a ERB através do canal de acesso. De posse destas duas grandezas podemos calcular a potência de transmissão no canal de tráfego da ERB, $P_{i}^{\text {traf }}$, a ser usada para que seja atingida a relação sinal-interferência $E_{b} / N_{t}$ desejada na ERM, ou seja [5]:

$$
\frac{E_{b}}{N_{t}}=\sum_{i=1}^{m} \frac{\frac{S_{i}^{\text {traf }}}{R_{b}}}{\frac{\left(\sum_{j=1}^{K} S_{j}^{\text {tot }}-\nu S_{i}^{\text {traf }}+N_{0} W\right)}{W}}
$$

onde $R_{b}$ é a taxa de bits, $W$ é a largura de banda do canal, $m$ é o número de ERBs envolvidas no processo de "soft-handoff" da ERM em questão, $S_{i}^{\text {traf }}$ é a potência de tráfego recebida pela ERM da $i$-ésima ERB, $S_{j}^{\text {tot }}$ é a potência total recebida da $j$-ésima ERB, $K$ é o número total de ERBs (células) no sistema e $N_{0}$ é a densidade espectral unilateral de potência do ruído branco.

A IS-95 [5] prevê dois tipos de controle de potência para o canal reverso: o de malha aberta e o de malha fechada. No primeiro instante, aplica-se o controle de malha aberta. Neste caso, a potência transmitida pela ERM é dada por [5]:

$$
P^{t o t}=-S^{t o t}-73+N O M_{-} P W R+I N I T_{-} P W R
$$

onde $N O M_{-} P W R$ é o fator de correção nominal para a ERB, enquanto que $I N I T \_P W R$ é o fator de correção para a ERM devido à descorrelação parcial na perda de percurso entre os enlaces direto e reverso, e $S^{\text {tot }}$ é a potência total recebida pela ERM, que é dada por [5]:

$$
\begin{aligned}
S^{\text {tot }} & =\sum_{j=1}^{L}\left[\left(P_{j}^{\text {pil }}+P_{j}^{s y n c}+P_{j}^{\text {pag }}+\nu \sum_{i=1}^{N_{j}} P_{i, j}^{\text {traf }}\right)\right. \\
& \left.\times L_{j} G_{b}^{j} G_{m}\right]+N_{0} W
\end{aligned}
$$

onde $P_{j}^{p i l}, P_{j}^{s y n c}, P_{j}^{p a g}$ são as potências transmitidas pela $j$ ésima ERB nos canais piloto, de sincronismo e de "paging", respectivamente, $P_{i, j}^{\text {traf }}$ é a potência transmitida no canal de tráfego pela $i$-ésima ERM da $j$-ésima célula, $N_{j}$ é o número de ERMs servidas pela $j$-ésima ERB, $L_{j}=r_{j}^{\beta}$ é a perda de percurso entre a ERM e a $j$-ésima ERB, e $G_{b}^{j}$ e $G_{m}$ são os ganhos de antena da $j$-ésima ERB e da ERM, respectivamente.

No processo de malha fechada, a ERB mede a relação sinal-interferência recebida do $i$-ésimo usuário e a compara com o valor desejado de $E_{b} / N_{t}$ no canal reverso para uma taxa de erro de quadro de $1 \%$. Caso esteja acima, a potência do móvel é reduzida, caso contrário, aumentada em passos de $P W R \_S T E P$. Esta comparação é atualizada em intervalos de $\Delta t$ segundos.

Toda ERM que desejar usar um canal de tráfego deve calcular a relação de energia de chip sobre a interferência total, proveniente de todas as ERBs do sistema, conforme [5]

$$
\left(\frac{E_{c}}{I_{0}}\right)=\frac{S_{i}^{p i l}}{N_{0} W+\sum_{j=1}^{K} S_{j}^{\text {tot }}}
$$

onde $S_{i}^{\text {pil }}$ é a potência recebida no canal piloto pela ERM devido à $i$-ésima ERB. Se a máxima relação $E_{c} / I_{0}$ recebida for menor do que $T_{A D D}$ (limiar de relação sinal-interferência pré-definido), esta chamada será bloqueada. Presume-se que a ERM encontra-se em uma região sombreada, ou em que a interferência recebida é muito alta. Caso contrário, a ERM estabelece um enlace rádio no canal reverso com a ERB. Neste caso a potência de transmissão será dada pelo controle de potência de malha aberta no canal reverso.

O estado de soft handoff de cada usuário é baseado na intensidade do sinal no canal piloto e é caracterizado pela classificação das ERBs em 3 conjuntos que são: ativo, candidato e restante. No conjunto ativo estão todas as ERBs que participam do processo de soft handoff, no candidato estão todas as ERBs que possuem condições de manter um canal de tráfego com a ERM e no conjunto restante, estão todas as ERBs que não fazem parte nem do conjunto ativo e nem do conjunto candidato. O processo de soft handoff pode ser resumido em apenas 3 passos:

1. Se a relação $E_{c} / I_{0}$ no canal piloto de alguma ERB no conjunto ativo ou candidato for menor do que $T_{D R O P}$ durante um tempo superior a $T_{T D R O P}$, então esta ERB é movida para o conjunto restante.

2. Se a relação $E_{c} / I_{0}$ recebida pelo canal piloto de alguma ERB no conjunto restante for maior do que $T_{A D D}$, então esta ERB é movida para o conjunto candidato.

3. Se a relação $E_{c} / I_{0}$ recebida pelo canal piloto de alguma ERB no conjunto candidato for maior do que a relação $E_{c} / I_{0}$ de alguma ERB no conjunto ativo de pelo menos $T_{C O M P}$, então a primeira ERB é movida para o conjunto ativo.

Se, durante o processo de "soft handoff", o conjunto ativo de alguma ERM ficar vazio (sem nenhuma ERB), esta ERM será desconectada do sistema, o que caracterizará uma queda de chamada.

Na implementação deste simulador, foi adotada uma abordagem temporal [6], que tem sido usada com sucesso para simulações de sistemas em tempo real, como é o caso de um sistema CDMA.

\section{RESULTADOS}

Com respeito à posição das ERBs, este software permite posicioná-las conforme qualquer tipo de agrupamento. Foi usado um agrupamento quadrado com 25 ERBs ao todo, com os parâmetros típicos definidos segundo a Tab. 1 .

Nas próximas oito figuras iremos ilustrar o comportamento de uma rede celular em relação ao desvio padrão do sombreamento, taxa de chegada de chamadas, expoente de perda de propagação, fator de atividade de voz, percentual de usuários em "soft-handoff", probabilidade de perda de desempenho, e taxa de bloqueio e de queda de chamadas bloqueadas.

A Fig. 1 nos mostra a capacidade de um sistema celular CDMA em termos do número médio de usuários por célula em função do desvio padrão do sombreamento, que foi variado entre 6 e $9 \mathrm{~dB}$, e da taxa de chegada de chamadas, que variou de 0,05 a 0,5 usuários/s/célula. Como a duração média das chamadas utilizada foi de $100 \mathrm{~s}$, esperava-se obter um número médio de ERMs por célula de $\lambda \mu$, que neste caso 


\begin{tabular}{||c|c|c||}
\hline \hline Parâmetro & Valor Padrão & Unidade \\
\hline$P_{j}^{\text {pil }}$ & 2 & $W$ \\
$P_{j}^{\text {sync }}$ & 0,2 & $W$ \\
$P_{j}^{\text {pag }}$ & 0,8 & $W$ \\
$G_{b}$ & 6 & $d B$ \\
$G_{m}$ & 0 & $d B$ \\
$N_{0}$ & -200 & $d B W / H z$ \\
$N O M_{-} P W R$ & 0 & $d B$ \\
$I N I T_{-} P W R$ & 0 & $d B$ \\
$W$ & 1,2288 & $M H z$ \\
$R_{b}$ & 9,6 & $k b / s$ \\
$T_{A D D}$ & -14 & $d B$ \\
$T_{D R O P}$ & -16 & $d B$ \\
$T_{C O M P}$ & 2,5 & $d B$ \\
$T_{T D R O P}$ & 5 & $s$ \\
$P_{\max }$ & 0,2 & $W$ \\
$\mu$ & 100 & $s$ \\
$v_{\min }$ & 0 & $k m / h$ \\
$v_{\max }$ & 100 & $k m / h$ \\
$P W R S T E P$ & 1 & $d B$ \\
$\left(E_{b} / N_{t}\right) r e v$ & 7 & $d B$ \\
$\left(E_{b} / N_{t}\right) d i r$ & 7 & $d B$ \\
\hline \hline
\end{tabular}

Tabella 1. Parâmetros do Simulador.

deveria variar entre 5 e 50 . Para um baixo valor de sombreamento e da taxa de chegada de chamadas o valor esperado foi obtido. À medida que a taxa de chegada e/ou o sombreamento cresce, a interferência também cresce, fazendo com que um número apreciável de ligações sejam bloqueadas ou derrubadas.

A Fig. 2 também nos mostra a capacidade em função do expoente da perda de propagação que foi variado entre 3 e 6 . Neste caso, o número médio de usuários por célula não varia $\operatorname{com} \beta$, pois a potência transmitida pelas ERMs é controlada, fazendo com que a interferência não cresça. Verificamos também para um alto valor da taxa de chegadas que o valor esperado obtido está longe do valor teórico, devido ao alto número de ligações bloqueadas e derrubadas.

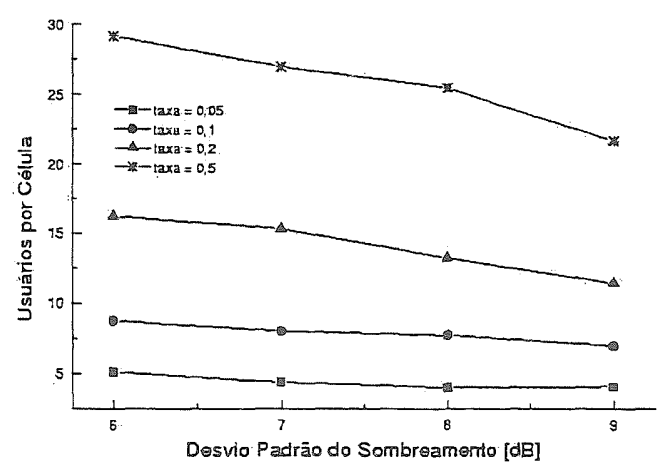

Figurra 1. Número Médio de Usuários por Célula em Função do Desvio Padrão do Sombreamento e da Taxa de Chegada de Chamadas para $\nu=0,4, \beta=4$ e $R=1700 \mathrm{~m}$.

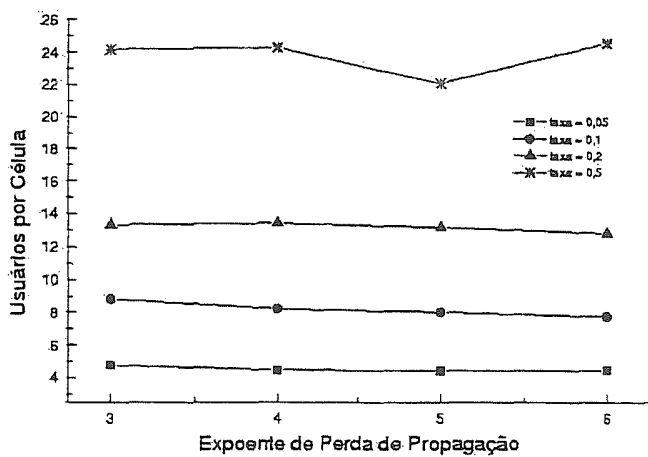

Figura 2. Número Médio de Usuários por Célula em Função do Expoente de Perda de Propagação e da Taxa de Chegada de Chamadas para $\nu=0,4, \sigma=8 \mathrm{~dB}$ e $R=1700 \mathrm{~m}$.

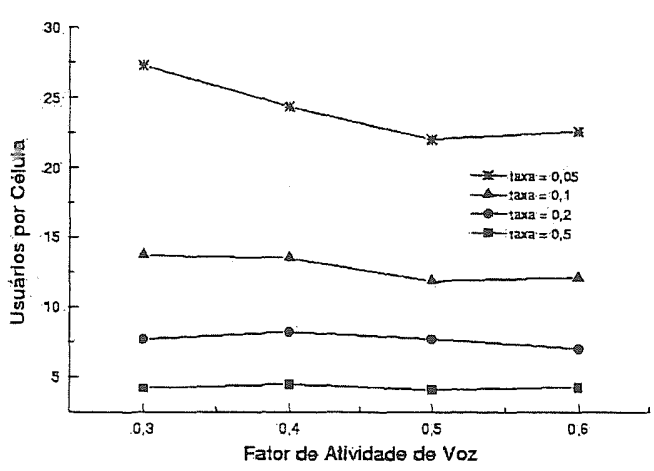

Figura 3. Número Médio de Usuários por Célula em Função do Fator de Atividade de Voz e da Taxa de Chegada de Chamadas para $\beta=4, \sigma=8 \mathrm{~dB}$ e $R=1700 \mathrm{~m}$.

A Fig. 3 nos mostra a capacidade desta vez em função do fator de atividade de voz que foi variado entre 0,3 e 0,6 . Neste caso, o número médio de usuários por célula não varia com $\nu$ para taxas de chegada de chamadas baixas, pois nesta situação o sistema está funcionando com folga. Por outro lado, para altas taxas de chegada de chamadas, quando o sistema funciona com muita interferência é que observa-se uma diminuição no número de usuários por célula.

As Fig. 4 e 5 apresentam o percentual de usuários instantâneo em processo de "soft-handoff" para uma dada taxa de chegada de chamadas. Podemos observar que, dependendo do tráfego, entre 12 e $25 \%$ dos usuários estão em processo de "soft-handoff". Podemos concluir também que o percentual de usuários em "soft-handoff" diminui com o aumento da taxa de chegada de chamadas, pois em altas taxas de chamadas um número apreciável de chamadas não será concluído.

A Fig. 6 nos mostra a probabilidade de perda de desempenho em função do limiar de relação sinal-interferência e da taxa de chegada de chamadas. A probabilidade de perda de desempenho nada mais é que a porcentagem de usuários com relação sinal-interferência no canal de tráfego abaixo da estipulada para uma taxa de erro de quadro menor que $1 \%$. 


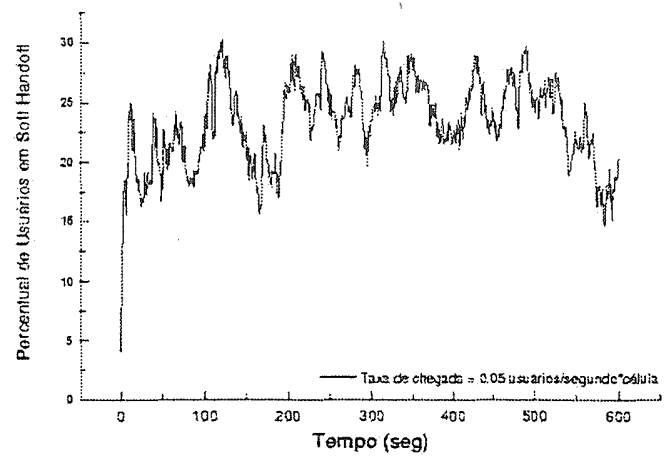

Figura 4. Percentual de Usuários em "Soft-Handoff" Instantâneo para $\lambda=0,05$ usuários $/ \mathrm{s} /$ célula, e $\nu=0,4, \sigma=8 \mathrm{~dB}$, $\beta=4$ e $R=1700 \mathrm{~m}$.

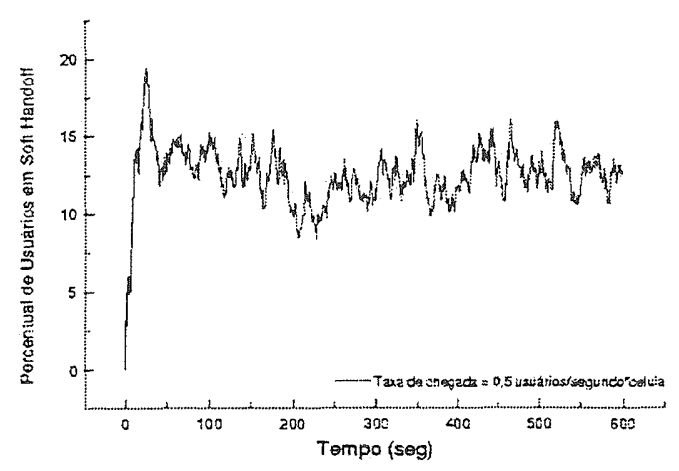

Figura 5. Percentual de Usuários em "Soft-Handoff" Instantâneo para $\lambda=0,5$ usuários/s/célula, e $\nu=0,4, \sigma=8 \mathrm{~dB}$, $\beta=4$ e $R=1700 \mathrm{~m}$.

Esta pode ser interpretada como a fração do tempo em que a relação sinal-interferência está abaixo de um certo limiar. Altas taxas de chegada de chamadas produzem, evidentemente, uma probabilidade de perda de desempenho maior para um dado limiar de relação sinal-ruído.

As Fig. 7 e 8 apresentam o percentual de bloqueio e de queda de chamadas em função do raio da célula em $\mathrm{m}$. Podemos observar na Fig. 7 que células com raio da ordem de $3 \mathrm{~km}$ apresentam um percentual de chamadas bloqueadas muito alto. O mesmo pode ser dito do percentual de queda de chamadas, observando-se a Fig. 8, para células com raio acima de $2 \mathrm{~km}$ e taxa de chegada de chamadas de 0,05 usuários/s/célula. Esta conclusão é válida para valores típicos dos parâmetros de uma rede CDMA, tais como fator de atividade de voz $\nu=0,4$, desvio padrão do sombreamento $\sigma=8 \mathrm{~dB}$ e expoente de propagação $\beta=4$.

\section{CONCLUSÃO}

Observamos o comportamento de uma rede celular CDMA em termos da capacidade, região de cobertura, probabilidade

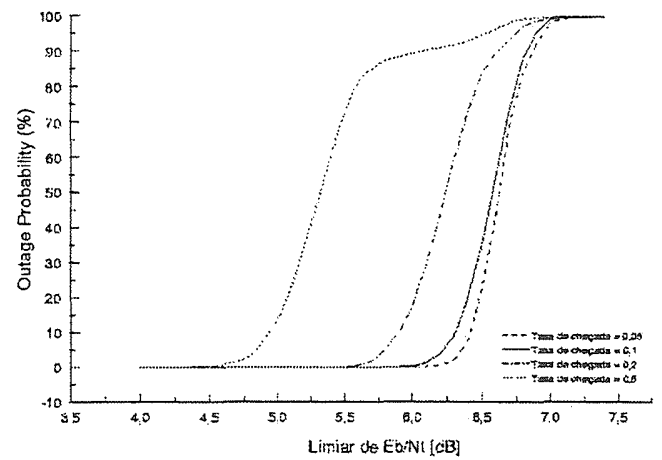

Figura 6. "Outage Probability" em Função do Limiar de Relação Sinal-Ruído e da Taxa de Chegada de Chamadas para $\nu=0,4, \sigma=8 \mathrm{~dB}, \beta=4$ e $R=1700 \mathrm{~m}$.

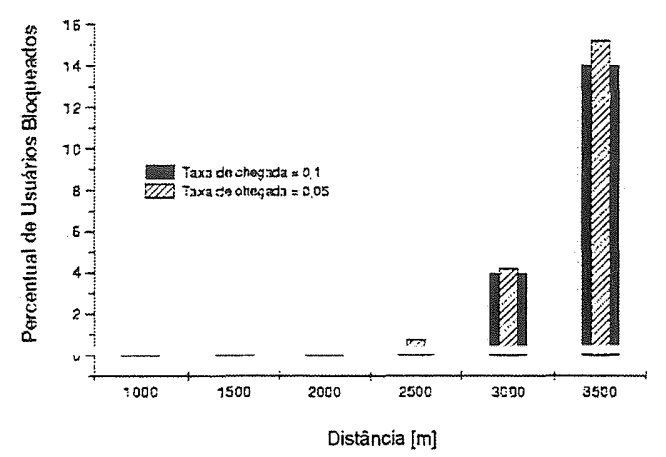

Figura 7. Percentual de Chamadas Bloqueadas em Função do Raio da Célula e da Taxa de Chegada de Chamadas para $\nu=0,4, \sigma=8 \mathrm{~dB}, \beta=4$.

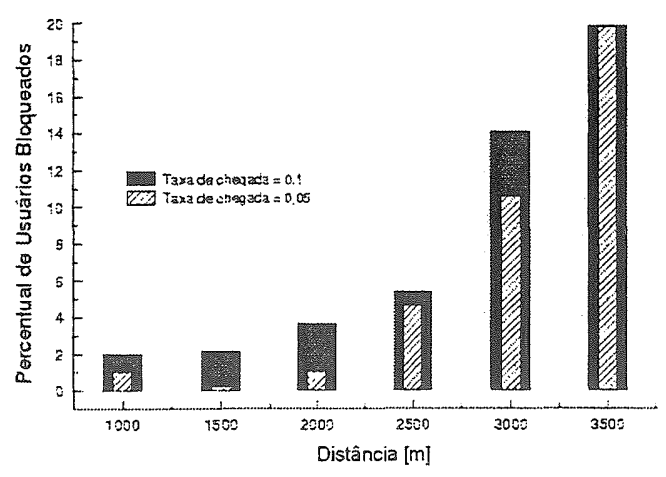

Figura 8. Percentual de Queda de Chamadas em Função do Raio da Célula e da Taxa de Chegada de Chamadas para $\nu=$ $0,4, \sigma=8 \mathrm{~dB}, \beta=4$. 
Luiz Gustavo Alarcon Alegro e Celso de Almeida Análise de Desempenho de uma Rede Celular CDMA através de Simulação

de perda de desempenho, percentual de bloqueio e de queda de chamadas, assim como do percentual de usuários em "softhandoff". Constatamos que para taxas de chegada de 0,05 usuários/s/célula, células com raio superior a $2 \mathrm{~km}$, podem apresentar alto percentual de queda de chamadas.

\section{REFERÊNCIAS}

[1] K. Pahlavan, A. H. Levesque. Wireless Information Networks. Wiley \& Sons, 1995.

[2] Seong-Lyun Kim, Seon-Ho Hwang, Chankyu Kim and Yougnam Han. A radio network simulator for IS-95 based CDMA systems: CDMA WiNS. IEEE Trans. Veh. Technology, pages 541-545, 1996.

[3] Szu-Wei Wang and Hua Mary Chion. Network simulations for IS-95 CDMA system. Proc. 5th WINLAB Workshop, pages 193-215, 1995.

[4] Luiz Gustavo Alarcon Alegro - Análise de Desempenho de uma Rede Celular CDMA Através de Simulação Tese de Mestrado - UNICAMP - 1998

[5] TIA/EIA/IS-95. Interim Standard - 95, July-1995.

[6] G. Gordon - System Simulation. Prentice-Hall, 2nd edition, 1978. 